\title{
Tapetal 3-Ketoacyl-Coenzyme A synthases are involved in pollen coat lipid accumulation for pollen-stigma interaction in Arabidopsis
}

\author{
Zaibao Zhang ${ }^{1}$, Huadong Zhan ${ }^{2}$, Jieyang $\mathrm{Lu}^{2}$, Shuangxi Xiong ${ }^{2}$, Naiying Yang $^{2}$, Hongyu \\ Yuan $^{1}$, and Zhong-Nan Yang ${ }^{2}$ \\ ${ }^{1}$ Xinyang Normal University \\ ${ }^{2}$ Shanghai Normal University
}

May 6, 2020

\begin{abstract}
Pollen coat lipids form an outer barrier to protect pollen itself and play essential roles in pollen-stigma interaction. However, the precise molecular mechanisms underlying pollen coat lipids production, deposition, regulation and function during anther development remain largely elusive. 3-ketoacyl-coenzyme A synthases (KCS) are involved in fatty acid elongation or very long chain fatty acid (VLCFA) synthesis. Here, we identified six members of Arabidopsis KCS family expressed in anther. Of them, KCS7, KCS15 and KCS21 express in tapetal cells at stages 8-10. Further analysis demonstrated that they act downstream of Male sterility1 (MS1), a regulator for late tapetum development. The kcs7/15/21 triple-mutant is fertile. Both cellular observation and lipid staining showed pollen coat lipid was decreased in triple mutant. After landing on stigma, the wild-type pollen was hydrated in about 5 min while the triple mutant pollen took about $10 \mathrm{~min}$. Pollen tube growth of the triple mutant was also delayed. These results demonstrate the pathway in tapetum to produce pollen coat lipid, and reveal the roles of tapetal-derived pollen coat lipid for pollen-stigma interaction.
\end{abstract}

\section{Summary Statement}

Pollen coat lipids play essential roles in pollen-stigma interaction. Mutation of tapetal-derived KCS proteins (KCS7, KCS15 and KCS21) affects pollen hydration and tube growth. A critical point of tapetal-derived lipids in pollen coat is required for pollen hydration.

Key words: pollen coat, lipid, 3-ketoacyl-coenzyme A synthase, pollen hydration INTRODUCTION

Pollen-pistil interaction is critical for the successful fertilization of flowering plants. This interaction is a crucial step in preventing inbreeding and maintaining species identity, thus contributing to angiosperm diversity. Pollen-pistil interaction consists of multiple selective steps, including pollen adhesion, hydration, germination and polarized tube growth (Bedinger, Broz, Tovar-Mendez, \& McClure, 2017; Edlund, Swanson, \& Preuss, 2004; Zheng, Lin, Liang, Wang, \& Chen, 2018). Pollen adhesion and hydration are two earliest events in pollination among species with dry stigmas. These highly regulated processes require proteins and lipids deriving from pollen wall (Elleman \& Dickinson, 1990; Safavian \& Goring, 2013).

The mature pollen wall of flowering plants includes three main layers, intine, exine and pollen coat. Intine is produced by microspores and is mainly composed of pectin, cellulose and hemicellulose. Exine is constituted of sexine and nexine (Lou et al., 2014). The main composition of sexine is sporopollenin. Its precursors are synthesized by multiple enzymes regulated by a MYB transcription factor MS188 in tapetum (K. Wang et al., 2018; Z. B. Zhang et al., 2007). Pollen coat contains many sticky and heterogenous material composed of lipids, proteins, carotenoids and polysaccharides (Hernández-Pinzón, Ross, Barnes, Damant, \& Murphy, 1999; 
Piffanelli, Ross, \& Murphy, 1998), which is crucial to pollen protection from abiotic stresses, successful pollen contact, hydration and subsequent pollen germination on dry stigma (Dickinson, 1995; Elleman \& Dickinson, 1990). The pollen coat contains various components including pollen coat proteins (PCP) and pollen coat lipids. PCPs are derived from tapetum after its programmed cell death (PCD) alongside gametophytically derived proteins. In Arabidopsis, several lipases and lipid-binding oleosin proteins were identified in PCPs (Mayfield, Fiebig, Johnstone, \& Preuss, 2001). Male sterility1 (MS1) is a PHD transcription factor for late tapetum development (Wilson, Morroll, Dawson, Swarup, \& Tighe, 2001). These sporophyte-derived PCPs are regulated by MS1 in tapetum (Lu et al., 2020). They may play roles in the early events of pollen-stigma recognition including pollen adhesion and hydration. S-locus cysteine-rich protein (SCR), SLR1-BP1/2, PCPA1(PCP7) and PCP-Bs have been identified as gametophyte-derived PCPs (Doughty et al., 1998; Nasrallah \& Nasrallah, 2014; Takayama et al., 2000; L. Wang et al., 2017). They are likely to play important roles in pollen germination and pollen tube growth.

Lipids are one of the major subcellular components, and comprise different combinations and positional distributions of fatty acids. They play important roles in plants by forming membrane structures, acting as storage lipids, signaling molecules, and surface coverings (Li-Beisson, Nakamura, \& Harwood, 2016). The pollen coat lipids display a semi-solid state and may guide the water transfer from the stigma to pollen during pollen-stigma interaction (Edlund et al., 2004). In lipid metabolism, 3-ketoacyl CoA synthase (KCS) are involved in fatty acid elongation or very long chain fatty acid (VLCFA) synthesis (Haslam \& Kunst, 2013; Joubès et al., 2008). Eceriferum 2 (Cer2) and cer2-like 2 (Cer2l2) encode BAHD acyltransferase and enhance the elongation of VLCFA from 28 to 30 carbon atoms catalyzed by KCS6 (Fiebig et al., 2000; Haslam et al., 2015). The kcs6 single mutant and cer2cer2l2 double mutant can produce mature pollen. However, they fail to hydrate (Fiebig et al., 2000; Haslam et al., 2015; Hulskamp, Schneitz, \& Pruitt, 1995; Preuss, Lemieux, Yen, \& Davis, 1993). KCS6, CER2 and CER2L2 express in endothecium suggesting that endothecium plays a role to synthesize pollen coat lipids for pollen hydration (Zhan, Xiong, Wang, \& Yang, 2018). It was generally considered that pollen coat lipids are derived from tapetum (Hernández-Pinzón et al., 1999). It is not clear how tapetum contributes to pollen coat lipids synthesis.

The KCS family in Arabidopsis contains 21 KCS members (Costaglioli et al., 2005; Joubès et al., 2008). In this study, we reported KCS7, KCS15 and KCS21 were expressed in tapetum. They act downstream of MS1, a regulator for late tapetum development. The reduced pollen coat lipid in the triple mutant $(k c s 7 / 15 / 21)$ and defective pollen hydration demonstrate that they play redundant roles for pollen coat lipid synthesis for pollen hydration.

\section{RESULTS}

\section{Six $K C S$ genes expressed in anther of Arabidopsis}

Arabidopsis genome contains $21 K C S$ genes. Of them, KCS1/5/6/7/9/10/11/13/15/20/21 were reported to express in flowers (Joubès et al., 2008). We performed reverse transcription-polymerase chain reaction (RT-PCR) analysis, and confirmed their expression in flowers (Fig. S1). Among them, KCS5 ,KCS7 and $K C S 15$ were specifically expressed in flowers, while KCS6, KCS9, KCS10, KCS13, KCS20 and KCS21 were relatively highly expressed in flowers (Fig. S1). To further understand the expression patterns of these KCS proteins in the developing anthers, the $K C S$ genomic sequences were fused to green fluorescent protein gene (GFP) to make the reporter constructs ( $p K C S:: K C S-G F P$ ) to transform WT Arabidopsis plants. Transgenic lines expressing $p K C S:: K C S$-GFP in anthers were identified (Fig.1a). The GFP signals of KCS7-GFP, KCS15GFP and KCS21-GFP proteins displayed similar expression patterns (Fig.1a). Their GFP fluorescence was detected in tapetum at anther stage 8-10 (Fig.1a). No GFP signals were detected at stage 11 when tapetum programmed cell death (PCD) occur. The expression of these $K C S$ s is quite similar with that of tapetal sporopollenin synthesis genes (K. Wang et al., 2018). KCS20 was expressed in tapetum at anther stage 9, and distributed within the anther locule and pollen at stage 9-11. KCS5-GFP signal was localized in epidermis at anther stages 10 to 12, while KCS10-GFP signal was preferentially detected in both epidermis and endothecium at anther stages 10 and 11 (Fig.1b). 


\section{$K C S 7, K C S 15$ and $K C S 21$ act downstream of $M S 1$}

Dysfunctional tapetum1 (DYT1), Defective in tapetal development and function 1 (TDF1), Aborted microspore (AMS), MS188, and MS1 are essential tapetum regulators which form a genetic pathway (Sorensen et al., 2003; Wilson et al., 2001; W. Zhang et al., 2006; Z. B. Zhang et al., 2007; Zhu et al., 2008; Zhu, Lou, Xu, \& Yang, 2011). Based on the microarray data, KCS7, KCS15 and KS21 act downstream of these regulators (Fig. 2a) (Zhu et al., 2011). We collected the inflorescences of dyt1 , tdf1 , ams ,ms188 and ms1 for quantitative real-time PCR analysis. These results showed that the expressions of KCS7, KCS15and KCS21 were downregulated while KCS20 was not affected in these mutants (Fig. 2b).

MS1 is the key regulator for late tapetum development in the genetic pathway (Zhu et al., 2011). The downregulation of KCS7, KCS15 and KCS21 in ms1 mutant suggested that these three KCS genes were regulated by MS1. To further analyze the molecular regulation between MS1 and KCS7, KCS15 and KCS21 , we introduced the $p K C S:: K C S$-GFP construct into the $m s 1$ plants and analyzed the distribution of KCSGFP signals (Fig. 1a). The GFP signals could not be detected in ms1 mutant while they were clearly observed in the tapetal cells of wild type (Fig. 1a). These results were consistent with the microarray and qPCR results (Fig. 2a,b), which further showed that KCS7, KCS15 and KCS21 act downstream of MS1 . MYB99 is a direct target of MS1 and its mutant displays a slight defect in pollen development (AlvesFerreira et al., 2007). The expressions ofKCS7, KCS15 and KCS21 were not changed inmyb99 (Fig. 2c). MS188 is an upstream regulator of MS1 (Zhu et al., 2011). Our recent work showed that many PCPs were downregulated in both $m s 188$ and $m s 1$, and expression of $M S 1$ driven by the MS188 promoter in $m s 188$ mutant ( $m s 188 / p M S 188:: M S 1$ ) restored the expression of PCPs (Lu et al., 2020). We performed RT-qPCR in the same transgenic line (ms188/pMS188::MS1), and the expressions of KCS7 and KCS15 were fully restored in $p M S 188:: M S 1$ transgenic plants (Fig. 2d). However, the expression of KCS21 did not show any recovery in $p M S 188:: M S 1$ transgenic plants compared with that inms1 (Fig. 2d).

\section{The pollen coat is reduced in $k c s 7 / 15 / 21$ triple mutant}

To investigate the functions of the KCS7, KCS15 andKCS21 in anther development, T-DNA insertion lines of KCS7 , KCS15 and KCS21 were identified (Fig. S2). These mutants exhibited drastically reduced expression of the relevant genes (Fig. S2). No obvious vegetative or reproductive morphological abnormalities were observed in any of the single (kcs7, kcs15, kcs21), double $(k c s 7 / 15, k c s 7 / 21, k c s 15 / k c s 21)$ and triple mutants of $K C S 7$, KCS15 and KCS21(kcs7/15/21, Fig. S3). Aborted pollen grains were occasionally observed in $k c s 15$ single mutant (70 of 1030), and the percentage of aborted pollen grains was increased to $13 \%$ inkcs $7 / k c s 15 / k c s 21$ triple mutant (110 of 853, Fig. S3).

Lipids were deposited in pollen coat during pollen development, and KCS proteins were responsible for lipid synthesis. To analysis whether mutation in $K C S 7, K C S 15$ and $K C S 21$ affect the pollen coat lipids, the scanning electron microscopy (SEM) and transmission electron microscope (TEM) observations were used. WT pollen grains displayed a well-organized exine and a smooth pollen coat. However, the well-smoothed pollen coat was disturbed, with many particles in the cavity of triple mutant pollen exine (Fig. 3). In addition, the severely defective pollen of $k c s^{7} / k c s 15 / k c s 21$ was small and displayed dis-organized exine covered with additional materials (Fig. 3). The diethyloxadicarbocyanine iodide $\left(\mathrm{DiOC}_{2}\right)$ was used to stain the fatty acid content of pollen wall and fatty acid exhibit a red fluorescence of $\mathrm{DiOC}_{2}$ staining (Gu et al., 2014). A strong red fluorescence of $\mathrm{DiOC}_{2}$ staining was observed in exine and pollen coat of the WT mature pollen (Fig. $3 \mathrm{~b}, \mathrm{c})$. Whereas, $k$ cs $^{7} / k c s 15 / k$ cs21 mature pollen showed weaker red signals with little or no signals observed in the pollen coat (Fig. 3b,c). Further TEM analysis showed $k c{ }^{7} / / k c s 15 / k c s 21$ pollen coat is disordered and thinner than the wild type (Fig. 3d,e,f,g). Therefore, these results indicate that lipid content may be reduced in the pollen coat of

$k c s 7 / k c s 15 / k c s 21$.

\section{Pollen hydration was delayed in $k c s 7 / 15 / 21$ triple mutant}

Pollen coat is generally considered to involve in pollen-stigma interaction. A pollen hydration assay was 
carried out to analyze if the reduced pollen coat in $k c s 7 / k c s 15 / k c s 21$ affect the pollen-stigma interaction. Ms188 is a male sterile line without any pollen inside anther while its stigmas are not affected (Z. B. Zhang et al., 2007). The pollen hydration assay was carried out by pollinating stigmas of $m s 188$ with pollen grains derived from WT and the $k c s 7 / 15 / 21$ triple mutant (Fig. 4). After landing on stigma, the wild-type pollen began to absorb water and became spherical (pollen hydration) in about 5 min (Fig. 4a). However, the hydration of thekcs7/15/21 triple mutant pollen occurred in 10 min after pollination. Accordingly, pollen germination of the triple mutant is also delayed (Fig. 4a). To determine if the hydration delay is derived from a defect in water absorption, an in vitro hydration of WT and $k c s^{7} / 15 / 21$ triple mutant pollen in PEG 3350 series was carried out (Fig. S4). The in vitro hydration was not significantly different between WT and $k c s^{7} 7 / 15 / 21$ triple mutant pollen in PEG 3350 series, indicating that the absence of KCS7, KCS15, and KCS21 proteins did not impair the ability of pollen to absorb water. To test whether the subsequent steps of pollination were also affected in $k c s^{\gamma} / 15 / 21$ triple mutant, we monitored pollen tube initiation and growth as previously described (Mayfield \& Preuss, 2000) (Fig. S5,4b). 20\% of WT pollen grains showed a pollen-tube emergence within 20 min of pollination, while, $k c s^{7} / 15 / 21$ triple mutant pollen-tube emergence was postponed to $30 \mathrm{~min}$ (Fig. 4c,S5). After $2 \mathrm{~h}$, WT pollen produced significantly longer tubes (470 um) and penetrated into the style, while pollen tubes of $k c s \% / 15 / 21$ triple mutant were much shorter (266 um) (Fig. S5). Therefore, the elongation of pollen tube was postponed in $k c s 7 / 15 / 21$ triple mutant (Fig. 4b,c,S5). Despite the observed defects in pollen hydration and pollen tube elongation in $k c s^{7} / 15 / 21$ triple mutant, there was no significant difference in seed set in $k c s 7 / 15 / 21$ triple mutant compared with WT plants.

The expression of KCS6 in endothecium persist much later than KCS7/15/21 in tapetum during anther development

Our recent work showed that KCS6 interacts with CER2/CER2L2 for pollen coat very-long-chain fatty acids (VLCFAs) synthesis required for pollen hydration (Zhan et al., 2018). Therefore, both KCS6 in endothecium and KCS7/15/21 in tapetum contribute to the synthesis of pollen coat lipids. Currently no efficient techniques are available to distinguish the difference of lipids synthesized by the tapetum and endothecium. We compared the expression of KCS and CER proteins during anther development to understand the accumulation of the lipids in pollen coat. In previous work, we obtained transgenic lines of $p$ KCS6::KCS6-GFP , pCER2::CER2GFP and $C$ CER2L2::CER2L2-GFP (Zhan et al., 2018). All the GFP transgenic plants were grown in the same pot and cultured in the same condition. The expression of KCS6, CER2 and CER2L2 displayed a long period in endothecium from anther stage 8 to 12 (Fig. 5a). KCS7, KCS15 and KCS21 were expressed in tapetum (stage 8 to 10) (Fig. 1,5). They displayed a shorter time period than KCS6, CER2 and CER2L2 (Fig. 5a). These results not only suggested the different functions between KCS7/15/21 and KCS6 in anther development, but also indicating the different roles of tapetum- and endothecium-derived lipids. Tapetumderived lipids may synthesize and deposited in pollen coat much earlier than endothecium-derived lipids. MS1 protein accumulates in tapetal cells at anther stage 7 and stage 8 (Yang, Vizcay-Barrena, Conner, \& Wilson, 2007), overlapping with that of KCS7, KCS15 and KCS21 (Fig. 5a), further indicating that the expression of KCS7, KCS15 andKCS21 were regulated by MS1 (Fig. 2).

\section{DISCUSSION}

\section{Pollen coat lipids derived from both tapetum and endothecium}

Pollen coat lipids are major part of pollen coat which constitutes the outer layer of pollen. Previous investigation suggests that some pollen coat lipids are derived from endothecium (Zhan et al., 2018). It was generally considered that pollen coat lipids are mainly derived from tapetum (Hernández-Pinzón et al., 1999). In this work, the reduced pollen coat lipids in the triple mutant of tapetum expressed genes $(K C S 7, K C S 15$ and KCS21 ) suggest that these KCS s play a role in tapetum to provide pollen coat lipids (Fig. 3, 5). KCS7, KCS15 and KCS21 were expressed in tapetum from stage 8 to 10 while tapetum programmed cell death (PCD) occurred after stage 10 (Fig. 1). It is likely that lipids begin to deposit to the outside of developing microspores before tapetum PCD. With tapetum PCD, all tapetum compounds including lipids deposit to the pollen coat. The expression of KCS6, CER2, and CER2L2 in endothecium is much later than the expression of KCS7, KCS15 and KCS21 in tapetum (Fig. 5a). This further supports the previous prediction that 
lipids from endothecium deposit outside of the lipids from tapetum (Zhan et al., 2018). KCSs catalyze the synthesis of C20 to C28 lipids, while CER2 and CER2L2 are required for the production of C28 to C34 lipids (Fiebig et al., 2000; Haslam et al., 2015). This indicates that the inner pollen coat lipid from tapetum is likely medium- and long-chain fatty acids ( $<28$ carbon atoms), while outer pollen coat lipid from endothecium is very-long-chain fatty acids ( $>30$ carbon atoms).

\section{Pollen coat lipids function in pollen-stigma interaction}

The pollen-stigma interaction includes pollen adhesion, hydration and pollen tube growth. The pollen coat lipids may display a semi-solid state and waterproof the pollen grain since from its dispersal until its capture on a compatible stigma (Wheeler, Franklin-Tong, \& Franklin, 2001). Following the pollen-stigma interactions, a 'foot-like' structure is established to enhance pollen-stigma adhesion (Elleman \& Dickinson, 1990). During 'foot' formation, the lipids may create a capillary system to facilitate water transfer from the stigma cell to desiccated pollen, and pollen hydration occurs. However, it is difficult to clearly separate the processes of pollen adhesion and hydration in most reports. The very-long-chain fatty acids from endothecium is predicted to locate outer pollen coat (Zhan et al., 2018). In the mutant of kcs6 andcer2cer2l2 , the pollen could not hydrate on stigma and the plants are male sterility (Fiebig et al., 2000; Haslam et al., 2015; Zhan et al., 2018). This hydration defects might result from adhesion defect. The delayed hydration in the triple mutant of $k c s 7 / 15 / 21$ suggest that the tapetum-derived lipids might assist in establishing a water gradient between the pollen and stigma after pollen adhesion (Fig. 4), and its mutation only postpone the pollen hydration (Fig. 5b). We propose that the endothecium-derived lipid might function early in pollen adhesion, while tapetum-derived lipid might function later in pollen hydration. This hypothesis need to be further studied. Hydration defects have also been reported in mutants of pollen coat proteins, such as EXL4, GRP17, PCP-B (Updegraff, Zhao, \& Preuss, 2009; L. Wang et al., 2017; Wolters-Arts, Lush, \& Mariani, 1998), indicating that lipids and proteins of pollen coat may work cooperatively to facilitate pollen hydration.

\section{The tapetum regulatory cascade for pollen coat lipid formation}

Tapetum provides nutrition for microspore development, secrets hydrolases for tetrad wall dissolution, and supply materials for pollen wall and pollen coat formation during anther development. In Arabidopsis, the genetic regulatory pathway DYT1-TDF1-AMS-MS188-MS1 is important for tapetum development and function (Gu et al., 2014; Lou et al., 2014; Lou et al., 2018; Zhu et al., 2011). In this pathway, AMS directly regulates ABCG26 for sporopollenin transportation (J. Xu et al., 2010) and MGT5 to provide the $\mathrm{Mg}^{+}$for microspores development (X. F. Xu et al., 2015). MS188 directly regulates CYP703A2 and other sporopollenin biosynthesis genes for sporopollenin synthesis and sexine formation (K. Wang et al., 2018; Xiong et al., 2016). Sexine is the outer pollen wall where pollen coat (including pollen coat lipids) are deposited. MS1 is a transcription factor for late tapetum development directly regulated by MS188. It regulates the expression of multiple pollen coat proteins (Lu et al., 2020). MS1 also regulates the expression of $K C S 7, K C S 15$ and $K C S 21$ for pollen coat lipid synthesis (Fig. 2, 5). This pollen coat lipid may provide a matrix for assembly of pollen coat PCPs (Fig. 5b). After tapetum PCD, the endothecium-derived lipids deposited outside of the pollen coat (Fig. 5b). Together, this work reveals that following outer pollen wall formation regulated by MS188, its directly regulating transcription factor MS1 regulates the downstream gene expression for pollen coat formation. This regulatory cascade is helpful to make sure that pollen wall and pollen coat are orderly synthesized during anther development.

\section{MATERIALS AND METHODS}

\section{Plant materials and growth conditions}

Arabidopsis thaliana plants were grown in greenhouse at $21^{\circ} \mathrm{C}$ and $16 \mathrm{~h}$ - light/8h-dark photoperiod. TDNA insertion mutant of $k c s 7$ (At1g71160, SALK_023400), kcs15 (At3g52160, SALK_209946C) andkcs21 (At5g49070, SALK_089611) in Col-0 background were all obtained from the ABRC stocks. T-DNA insertion status of $K C S 7, K C S 15$ and KCS21 were confirmed using the T-DNA border primer SALK LBb1.3 in combination with gene-specific primers listed in Table S1. 


\section{RNA extraction and RT-qPCR}

Total RNA was isolated using TRIzol reagent (Invitrogen) according to the manufacturer's instructions. RNA quantity and purity were assessed with NanoQuant spectrophotometer. First strand cDNAs were synthesized from DNase I-treated total RNA using cDNA Synthesis Kit (Takara). Gene expression was normalized using tubulin (At5g23860) as the reference gene, and relative gene expression was calculated as the mean of three biological replicates and three technical replicates. Gene specific primers used for RT-qPCR are listed in Table S1.

\section{Microscopy and phenotypic characterization}

Pollen viability was assessed using Alexander's staining. For scanning electron microscopic (SEM) analysis, mature pollen grains from freshly dehisced anthers were mounted and coated with gold on stubs. The samples were then immediately observed using FESEM (SU8010). TEM (transmission electron microscopy) analysis was performed as described previously (Z. B. Zhang et al., 2007). The procedure of semi-thin section and lipid staining were performed as described previously (Lou et al., 2014; Z. B. Zhang et al., 2007).

\section{Protein localization analysis}

The transgenic plants of KCS5-GFP, KCS7-GFP, KCS10-GFP, KCS15-GFP, KCS20-GFP and KCS21GFP were constructed by fusing GFP with genomic sequences of KCS5, KCS7, KCS10, KCS15 , KCS20 and KCS21 driven by their native promoters. The flower buds of the transgenic plants were harvested and the anthers were isolated. The isolated anthers were scanned without fixation with Zeiss LSM510 confocal scanning microscope.

\section{Semi-in vivo pollen hydration and pollen tube growth assay}

For pollen hydration, freshly opened $m s 188$ pistil was cut and attached upright on agar (1.0\%). Then pollen grains $\left(\mathrm{n}=50^{\sim} 80\right)$ from a freshly dehiscing anther were transferred onto the stigmatic papilla. The behavior of the pollen was captured under a microscope immediately after pollinations were initiated. Forin vivo pollen tube growth analysis, pollinations were initiated on $m s 188$ stigmas and allowed to proceed for 2 $\mathrm{h}$ as described previously (L. Wang et al., 2017). Then stigmas were fixated overnight $(60 \% \mathrm{v} / \mathrm{v}$ ethanol, $30 \% \mathrm{v} / \mathrm{v}$ chloroform, $10 \% \mathrm{v} / \mathrm{v}$ acetic acid), incubated in $8 \mathrm{M} \mathrm{NaOH}$ for $20 \mathrm{~min}$, and washed in $\mathrm{dH}_{2} \mathrm{O}$ three times $(5 \mathrm{~min} / \mathrm{each})$. Samples were dyed in $0.1 \%$ decolourized aniline blue $(0.1 \% \mathrm{w} / \mathrm{v}$ aniline blue in $0.1 \mathrm{M}$ $\mathrm{K}_{3} \mathrm{PO}_{4}, \mathrm{pH} 11$ ) for $1 \mathrm{~h}$ and images were captured with an Olympus BX51 microscope. Pollen tube growth was calculated as the mean of three biological replicates. All statistical analyses were carried out using Microsoft Excel.

\section{ACKNOWLEDGMENTS}

This work was supported by the grants from National Natural Science Foundation of China (31870296, 31700277), Natural Science Foundation of Henan (No. 182300410063), Nanhu Scholars Program for Young Scholars of Xinyang Normal University, the Shanghai Science and Technology Committee (18DZ2260500, 17DZ2252700), and the Collaborative Innovation Center for Modern Crop Production to H.Z.

\section{Author contributions}

Z.Y., Z.Z. and H.Z. planned and designed the research and wrote the manuscript; Z.Z. and H.Z. were involved with all aspects of the research with S.X., J.L. and N.Y. contributing to expression analysis, SEM and TEM work; H.Y. assisted with critical assessment of the manuscript.

\section{Figure legend}

Figure 1. Expression analysis of KCS proteins during anther development. a, The expression of KCS7-GFP, KCS15-GFP and KCS21-GFP fusion proteins were initially detected in tapetum at stage 8 and subsequently accumulated in tapetum and anther locule at stages 9 and 10. After stage 10, the GFP signal was disappeared. The expression of KCS20-GFP fusion protein was initially detected in tapetum at stage 9 and subsequently accumulated in anther locule and pollen at stages 10 and 11 . GFP signal in tapetum was 
shown in enlarged view. No GFP signals of KCS7-GFP, KCS15-GFP and KCS21-GFP were identified in $m s 1$ background. T, tapetum. Bars $=20 \mu \mathrm{m}$. b, The expression of KCS10-GFP fusion protein was detected in epidermis and endothecium at stage 10 and stage 11, while the GFP signal of KCS5 was only detected in epidermis from anther stage 10 to 12 . Bars $=10 \mu \mathrm{m}$.

Figure 2. MS1 regulates the expression of $K C S 7, K C S 15$ and KCS21. a, Heatmap showing the expression of $K C S 7, K C S 15$, and KCS21 genes were downregulated in $d y t 1$, $t d f 1$, ams, $m s 188$, and $m s 1$ mutants, while the expression of $K C S 20$ was not affected in these mutants. b, qPCR analysis of $K C S$ genes indyt1, tdf1, ams, ms188, and ms1 mutants. c, RT-PCR analysis of KCS7, KCS15, and KCS21 genes in ms1,and myb99 mutants. d, Expression of KCS7, KCS15 and KCS21 genes was analyzed in pMS188::MS1 transgenic plants in the ms188 homozygous background (rescue).

Figure 3. Pollen coat was affected inkcs7/15/21 triple mutant. a, Scanning electron microscopic (SEM) analysis of exine and pollen coat morphology in WT and $k c s 7 / 15 / 21$ triple mutant plants. Red arrows and blue arrows indicated irregular pollen coat and particles, respectively. b and c, Lipid staining of semi-thin sections of WT andkcs7 / 15 / 21 triple mutant plants at anther stage 12. The red fluorescence indicates the lipid staining on mature pollen grains (white arrow). Bars $=10 \mu \mathrm{m} . \mathrm{d}, \mathrm{e}, \mathrm{f}$ and $\mathrm{g}$, Analysis of pollen wall structure by transmission electron microscopy (TEM). The ultrastructure of the pollen coat of $k c s 7 / 15$ / 21 triple mutant showed decreased and disordered compared with the wild type. Bars $=5 \mu \mathrm{m}$ in $\mathrm{d}$, e, and bars $=1 \mu \mathrm{m}$ in $\mathrm{f}$, g.

Figure 4. In vivo pollen hydration and pollen tube growth analysis in $k c s 7 / 15 / 21$ triple mutant. a, Diagram of pollen hydration in WT and $k c s^{7} / 15 / 21$ triple mutant. b, Diagram of pollen germination on stigma. c, In vivopollen tube emergence after manual pollination at $10 \mathrm{~min}, 20 \mathrm{~min}, 30 \mathrm{~min}$, $45 \mathrm{~min}, 1 \mathrm{~h}$ and $2 \mathrm{~h}$ post-pollination. Pollen tubes were emerged in WT pollen after manual pollination at $20 \mathrm{~min}$, while pollen tubes were identified in $k \mathrm{cs}^{7} / 15 / 21$ triple mutant at $30 \mathrm{~min}$. Pollen grains were applied to stigmas of $m s 188$. Bars $=10 \mu \mathrm{m}$.

Figure 5. A proposal model for anther derived lipids in pollen wall development. a, Expression pattern of KCS6, KCS15, CER2, and CER2L2 proteins during anther development. The expression of KCS15-GFP was detected in tapetum, while the expression of KCS6-GFP, CER2-GFP and CER2L2-GFP fusion proteins were detected in endothecium. Bars $=20 \mu \mathrm{m}$. b, A proposed model for tapetum and endothecium in biosynthesis of lipids for pollen wall development. In tapetum, MS1 regulates the expression of $K C S^{7} 7$ , KCS15 and KSC21, and the KCS-mediated lipids were deposited in pollen coat. In endothecium, CER2 and CER2L2 interact with KCS6 to modulate the biosynthesis of pollen coat VLCFAs for pollen hydration.

\section{Supplement Data}

Figure S1. Reverse transcription polymerase chain reaction (RT-PCR) expression analysis of eleven $K C S$ genes in root, stem, leaf and flowers.

Figure S2. RT-PCR analysis of $k c s 7, k c s 15, k c s 21$ single mutants and $k c s 7 / 15 / 21$ triple mutant.

Figure S3. Phenotypes of single, double and triple mutants ofkcs7, $k c s 15$ and $k c s 21$. a and b, Plants, anthers and pollen grains of $k c s 7, k c s 15, k c s 21$ single mutants and $k c s^{7} / 15$ / 21 triple mutant. Black arrows indicated aborted pollen grains in $k c s 15$ andkcs $7 / 15 / 21$ triple mutants. Bars $=20 \mu \mathrm{m}$. c, Cell biological analyses of WT and $k c s^{7} / 15 / 21$ triple mutant anthers using semi-thin transverse sections. Bars= $20 \mu \mathrm{m}$. d, Pollen grains from the single, double and triple mutants of the KCS7, KCS15 and KCS21 genes visualized under scanning electron microscope. Scale bar for huge pollen $50 \mu \mathrm{m}$; for single pollen $10 \mu \mathrm{m}$.

Figure S4. Width of $k c s 7 / 15$ / 21 triple mutant and wild-type pollen when placed in a PEG 3350 series. Each point represents the average width of 100 pollen grains.

Figure S5. $k c s 7 / 15$ / 21 triple mutant and wild-type in vivo pollen tube growth. a, Emergence of pollen tube with aniline blue staining. Scale bar represents $10 \mathrm{um}$ in figures of 10, 20, 30 and $45 \mathrm{~min}, 80 \mathrm{um}$ in figure of 1 hour, $100 \mathrm{um}$ in figure of 2 hour. b, The length of pollen tubes at different hours. 
Supplemental Table 1. Primers used in this study

\section{References}

Alves-Ferreira, M., Wellmer, F., Banhara, A., Kumar, V., Riechmann, J. L., \& Meyerowitz, E. M. (2007). Global expression profiling applied to the analysis of Arabidopsis stamen development. Plant Physiol, 145 (3), 747-762. doi:10.1104/pp.107.104422

Bedinger, P. A., Broz, A. K., Tovar-Mendez, A., \& McClure, B. (2017). Pollen-Pistil Interactions and Their Role in Mate Selection. Plant Physiol, 173 (1), 79-90. doi:10.1104/pp.16.01286

Costaglioli, P., Joubès, J., Garcia, C., Stef, M., Arveiler, B., Lessire, R., \& Garbay, B. (2005). Profiling candidate genes involved in wax biosynthesis in Arabidopsis thaliana by microarray analysis. Biochim Biophys Acta, 1734 (3), 247-258. doi:10.1016/j.bbalip.2005.04.002

Dickinson, H. (1995). Dry stigmas, water and self-incompatibility inBrassica. Sexual Plant Reproduction, 8 (1), 1-10.

Doughty, J., Dixon, S., Hiscock, S. J., Willis, A. C., Parkin, I. A., \& Dickinson, H. G. (1998). PCP-A1, a defensin-like Brassica pollen coat protein that binds the S locus glycoprotein, is the product of gametophytic gene expression. Plant Cell, 10 (8), 1333-1347. doi:10.1105/tpc.10.8.1333

Edlund, A. F., Swanson, R., \& Preuss, D. (2004). Pollen and stigma structure and function: the role of diversity in pollination.Plant Cell, 16 Suppl (Suppl), S84-97. doi:10.1105/tpc.015800

Elleman, C. J., \& Dickinson, H. G. (1990). The role of the exine coating in pollen-stigma interactions in Brassica oleracea L. New Phytologist, 114 (3), 511-518.

Fiebig, A., Mayfield, J. A., Miley, N. L., Chau, S., Fischer, R. L., \& Preuss, D. (2000). Alterations in CER6, a gene identical to CUT1, differentially affect long-chain lipid content on the surface of pollen and stems. Plant Cell, 12 (10), 2001-2008. doi:10.1105/tpc.12.10.2001

Gu, J. N., Zhu, J., Yu, Y., Teng, X. D., Lou, Y., Xu, X. F., . . Yang, Z. N. (2014). DYT1 directly regulates the expression of TDF1 for tapetum development and pollen wall formation in Arabidopsis. Plant J, 80 (6), 1005-1013. doi:10.1111/tpj.12694

Haslam, T. M., Haslam, R., Thoraval, D., Pascal, S., Delude, C., Domergue, F., . . . Joubès, J. (2015). ECERIFERUM2-LIKE proteins have unique biochemical and physiological functions in very-long-chain fatty acid elongation. Plant Physiol, 167 (3), 682-692. doi:10.1104/pp.114.253195

Haslam, T. M., \& Kunst, L. (2013). Extending the story of very-long-chain fatty acid elongation. Plant Sci, 210 , 93-107. doi:10.1016/j.plantsci.2013.05.008

Hernández-Pinzón, I., Ross, J. H., Barnes, K. A., Damant, A. P., \& Murphy, D. J. (1999). Composition and role of tapetal lipid bodies in the biogenesis of the pollen coat of Brassica napus. Planta, 208 (4), 588-598. doi:10.1007/s004250050597

Hulskamp, M., Schneitz, K., \& Pruitt, R. E. (1995). Genetic Evidence for a Long-Range Activity That Directs Pollen Tube Guidance in Arabidopsis. Plant Cell, 7 (1), 57-64. doi:10.1105/tpc.7.1.57

Joubès, J., Raffaele, S., Bourdenx, B., Garcia, C., Laroche-Traineau, J., Moreau, P., . . Lessire, R. (2008). The VLCFA elongase gene family in Arabidopsis thaliana: phylogenetic analysis, 3D modelling and expression profiling. Plant Mol Biol, 67 (5), 547-566. doi:10.1007/s11103-008-9339-z

Li-Beisson, Y., Nakamura, Y., \& Harwood, J. (2016). Lipids: From Chemical Structures, Biosynthesis, and Analyses to Industrial Applications. Subcell Biochem, 86 , 1-18. doi:10.1007/978-3-319-25979-6_1

Lou, Y., Xu, X. F., Zhu, J., Gu, J. N., Blackmore, S., \& Yang, Z. N. (2014). The tapetal AHL family protein TEK determines nexine formation in the pollen wall. Nat Commun, 5 , 3855. doi:10.1038/ncomms4855 
Lou, Y., Zhou, H. S., Han, Y., Zeng, Q. Y., Zhu, J., \& Yang, Z. N. (2018). Positive regulation of AMS by TDF1 and the formation of a TDF1-AMS complex are required for anther development in Arabidopsis thaliana. New Phytol, 217 (1), 378-391. doi:10.1111/nph.14790

Lu, J. Y., Xiong, S. X., Yin, W. Z., Teng, X. D., Lou, Y., Zhu J., Zhang, C., Gu, J. N., Wilson, Z. A., Yang, Z. N. (2020). MS1, a direct target of MS188, regulates the expression of key sporophytic pollen coat protein genes in Arabidopsis. J Exp Bot, (DOI: 10.1093/jxb/eraa219).

Mayfield, J. A., Fiebig, A., Johnstone, S. E., \& Preuss, D. (2001). Gene families from the Arabidopsis thaliana pollen coat proteome.Science, 292 (5526), 2482-2485. doi:10.1126/science.1060972

Mayfield, J. A., \& Preuss, D. (2000). Rapid initiation of Arabidopsis pollination requires the oleosin-domain protein GRP17. Nat Cell Biol, 2 (2), 128-130. doi:10.1038/35000084

Nasrallah, J. B., \& Nasrallah, M. E. (2014). S-locus receptor kinase signalling. Biochem Soc Trans, 42 (2), 313-319. doi:10.1042/bst20130222

Piffanelli, P., Ross, J. H., \& Murphy, D. J. (1998). Biogenesis and function of the lipidic structures of pollen grains. Sexual Plant Reproduction, 11 (2), 65-80. doi:10.1007/s004970050122

Preuss, D., Lemieux, B., Yen, G., \& Davis, R. W. (1993). A conditional sterile mutation eliminates surface components from Arabidopsis pollen and disrupts cell signaling during fertilization. Genes Dev, 7 (6), 974985. doi:10.1101/gad.7.6.974

Safavian, D., \& Goring, D. R. (2013). Secretory activity is rapidly induced in stigmatic papillae by compatible pollen, but inhibited for self-incompatible pollen in the Brassicaceae. PLoS One, 8 (12), e84286. doi:10.1371/journal.pone.0084286

Sorensen, A. M., Kröber, S., Unte, U. S., Huijser, P., Dekker, K., \& Saedler, H. (2003). The Arabidopsis ABORTED MICROSPORES (AMS) gene encodes a MYC class transcription factor. Plant J, 33 (2), 413-423. doi:10.1046/j.1365-313x.2003.01644.x

Takayama, S., Shiba, H., Iwano, M., Asano, K., Hara, M., Che, F. S., . . Isogai, A. (2000). Isolation and characterization of pollen coat proteins of Brassica campestris that interact with S locus-related glycoprotein 1 involved in pollen-stigma adhesion. Proc Natl Acad Sci U S A, 97 (7), 3765-3770. doi:10.1073/pnas.040580797

Updegraff, E. P., Zhao, F., \& Preuss, D. (2009). The extracellular lipase EXL4 is required for efficient hydration of Arabidopsis pollen.Sex Plant Reprod, 22 (3), 197-204. doi:10.1007/s00497-009-0104-5

Wang, K., Guo, Z. L., Zhou, W. T., Zhang, C., Zhang, Z. Y., Lou, Y., . . . Yang, Z. N. (2018). The Regulation of Sporopollenin Biosynthesis Genes for Rapid Pollen Wall Formation. Plant Physiol, 178 (1), 283-294. doi:10.1104/pp.18.00219

Wang, L., Clarke, L. A., Eason, R. J., Parker, C. C., Qi, B., Scott, R. J., \& Doughty, J. (2017). PCP-B class pollen coat proteins are key regulators of the hydration checkpoint in Arabidopsis thaliana pollen-stigma interactions. New Phytol, 213 (2), 764-777. doi:10.1111/nph.14162

Wheeler, M. J., Franklin-Tong, V. E., \& Franklin, F. C. H. (2001). The molecular and genetic basis of pollen-pistil interactions. New Phytol, 151 (3), 565-584. doi:10.1046/j.0028-646x.2001.00229.x

Wilson, Z. A., Morroll, S. M., Dawson, J., Swarup, R., \& Tighe, P. J. (2001). The Arabidopsis MALE STERILITY1 (MS1) gene is a transcriptional regulator of male gametogenesis, with homology to the PHDfinger family of transcription factors. Plant J, 28 (1), 27-39. doi:10.1046/j.1365-313x.2001.01125.x

Wolters-Arts, M., Lush, W. M., \& Mariani, C. (1998). Lipids are required for directional pollen-tube growth. Nature, 392 (6678), 818-821. doi:10.1038/33929

Xiong, S. X., Lu, J. Y., Lou, Y., Teng, X. D., Gu, J. N., Zhang, C., . . Zhu, J. (2016). The transcription factors MS188 and AMS form a complex to activate the expression of CYP703A2 for sporopollenin biosynthesis in 
Arabidopsis thaliana. Plant J, 88 (6), 936-946. doi:10.1111/tpj.13284

Xu, J., Yang, C., Yuan, Z., Zhang, D., Gondwe, M. Y., Ding, Z., . . Wilson, Z. A. (2010). The ABORTED MICROSPORES regulatory network is required for postmeiotic male reproductive development in Arabidopsis thaliana. Plant Cell, 22 (1), 91-107. doi:10.1105/tpc.109.071803

Xu, X. F., Wang, B., Lou, Y., Han, W. J., Lu, J. Y., Li, D. D., . . Yang, Z. N. (2015). Magnesium Transporter 5 plays an important role in $\mathrm{Mg}$ transport for male gametophyte development in Arabidopsis.Plant J, 84 (5), 925-936. doi:10.1111/tpj.13054

Yang, C., Vizcay-Barrena, G., Conner, K., \& Wilson, Z. A. (2007). MALE STERILITY1 is required for tapetal development and pollen wall biosynthesis. Plant Cell, 19 (11), 3530-3548. doi:10.1105/tpc.107.054981

Zhan, H., Xiong, H., Wang, S., \& Yang, Z. N. (2018). Anther Endothecium-Derived Very-LongChain Fatty Acids Facilitate Pollen Hydration in Arabidopsis. Mol Plant, 11 (8), 1101-1104. doi:10.1016/j.molp.2018.05.002

Zhang, W., Sun, Y., Timofejeva, L., Chen, C., Grossniklaus, U., \& Ma, H. (2006). Regulation of Arabidopsis tapetum development and function by DYSFUNCTIONAL TAPETUM1 (DYT1) encoding a putative bHLH transcription factor. Development, 133 (16), 3085-3095. doi:10.1242/dev.02463

Zhang, Z. B., Zhu, J., Gao, J. F., Wang, C., Li, H., Li, H., . . Yang, Z. N. (2007). Transcription factor AtMYB103 is required for anther development by regulating tapetum development, callose dissolution and exine formation in Arabidopsis. Plant J, 52 (3), 528-538. doi:10.1111/j.1365-313X.2007.03254.x

Zheng, Y. Y., Lin, X. J., Liang, H. M., Wang, F. F., \& Chen, L. Y. (2018). The Long Journey of Pollen Tube in the Pistil. Int J Mol Sci, 19 (11). doi:10.3390/ijms19113529

Zhu, J., Chen, H., Li, H., Gao, J. F., Jiang, H., Wang, C., . . Yang, Z. N. (2008). Defective in Tapetal development and function 1 is essential for anther development and tapetal function for microspore maturation in Arabidopsis. Plant J, 55 (2), 266-277. doi:10.1111/j.1365-313X.2008.03500.x

Zhu, J., Lou, Y., Xu, X., \& Yang, Z. N. (2011). A genetic pathway for tapetum development and function in Arabidopsis. J Integr Plant Biol, 53 (11), 892-900. doi:10.1111/j.1744-7909.2011.01078.x

\section{Hosted file}

Fig 1.pdf available at https://authorea.com/users/318155/articles/448089-tapetal-3-ketoacylcoenzyme-a-synthases-are-involved-in-pollen-coat-lipid-accumulation-for-pollen-stigmainteraction-in-arabidopsis 
(a)

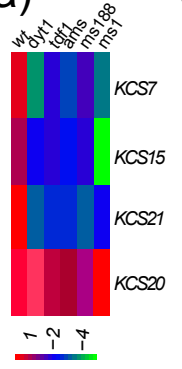

(b)

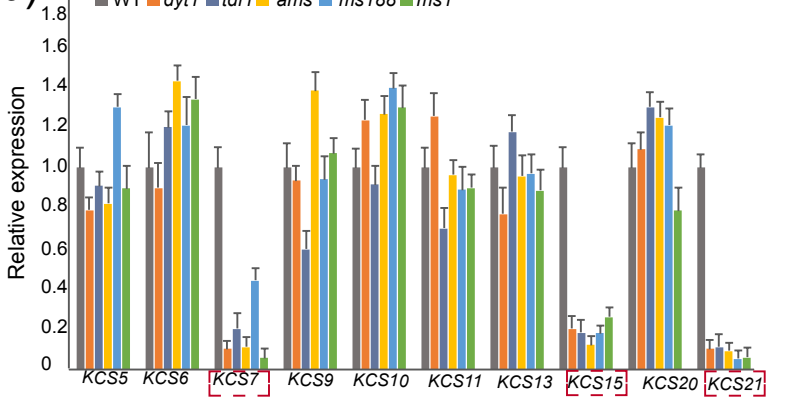

(c)

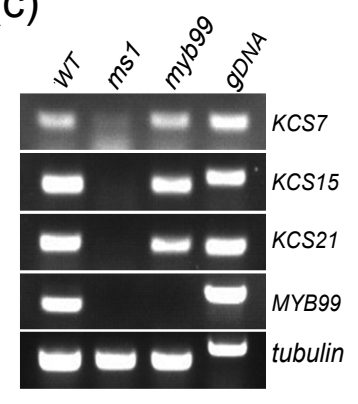

(d)

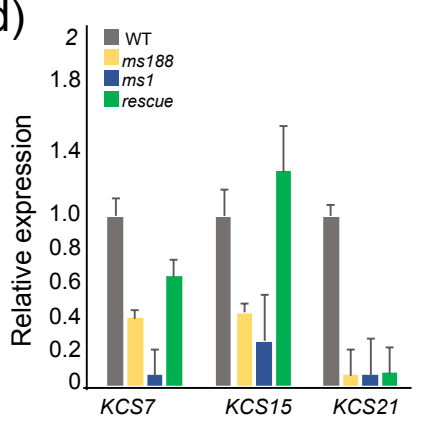




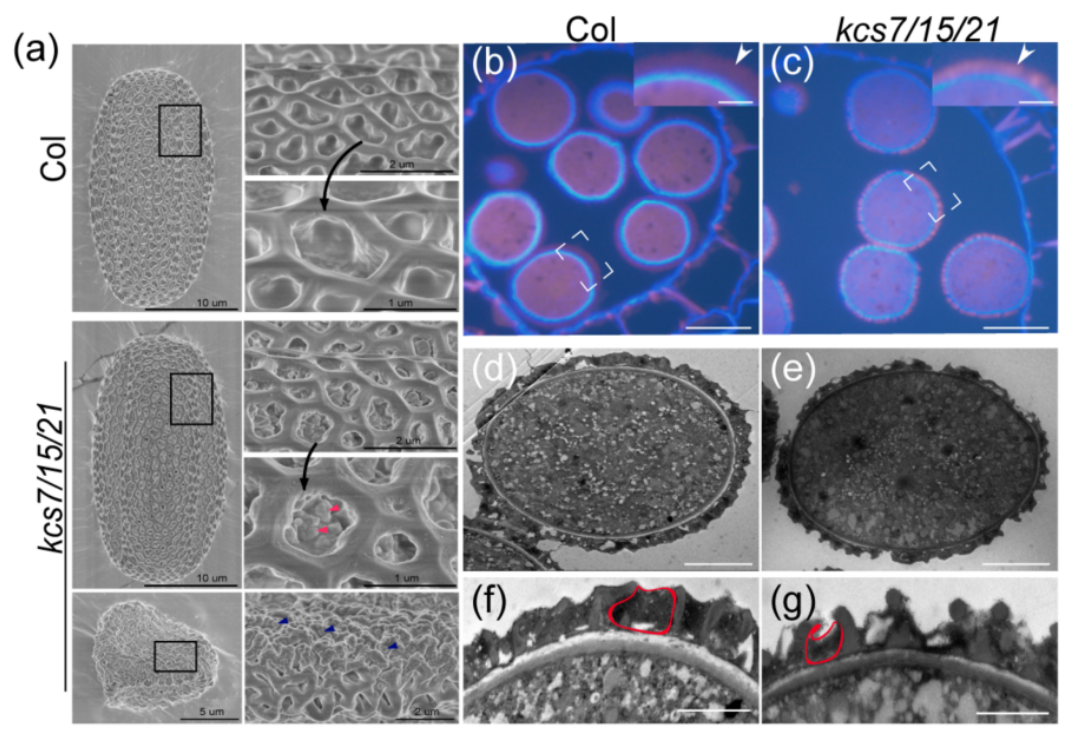


(a)

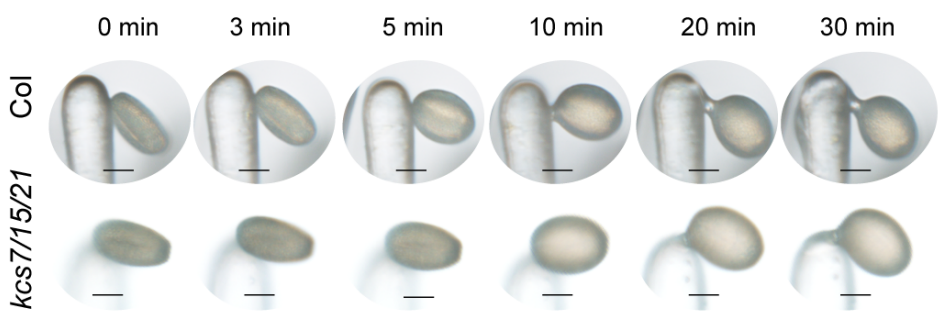

(b)
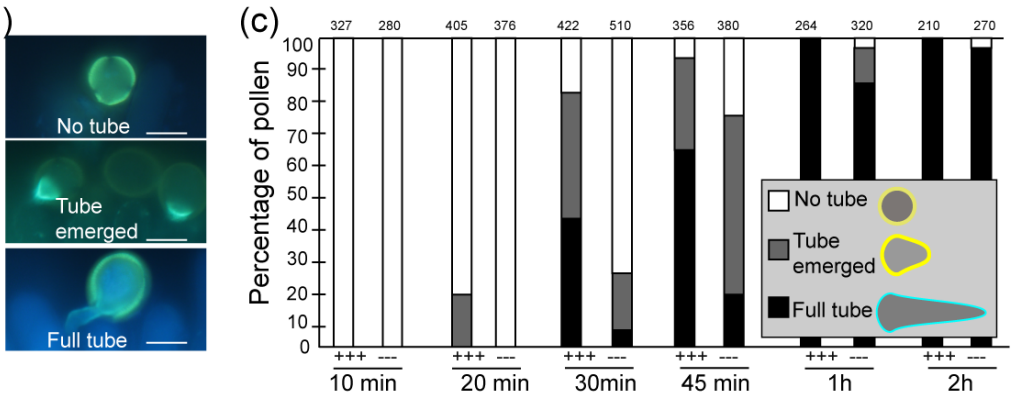

\section{Hosted file}

Fig 5.pdf available at https://authorea.com/users/318155/articles/448089-tapetal-3-ketoacylcoenzyme-a-synthases-are-involved-in-pollen-coat-lipid-accumulation-for-pollen-stigmainteraction-in-arabidopsis 